\title{
RETRACTED ARTICLE: Interference of Mucin 1 inhibits the growth of liver cancer cells by inducing mitochondria-mediated and death receptor-mediated cell apoptosis
}

\author{
Yongzhen Zhai • Deping Ding • Liya Wei • Enjing Chen • \\ Guohe Feng
}

Received: 9 May 2014 / Accepted: 19 May 2014 / Published online: 6 June 2014

(C) International Society of Oncology and BioMarkers (ISOBM) 2015

This article has been retracted due to the authors wish to retract this article "Interference of Mucin1 inhibits the growth of liver cancer cells by inducing mitochondria mediated and death receptor mediated cell apoptosis" by Yongzhen Zhai, Deping Ding, Liya Wei, Enjing Chen, Guohe Feng, published in Tumor Biology, 2014, DOI: 10.1007/s13277-014-2120-9. Concerns were raised on the accuracy of the data presented and as consequence in the results and conclusions of the article for which the authors accept full responsibility. The authors apologize to the readers, reviewers, and editors for publishing this erroneous data.

\footnotetext{
This article has been retracted due to the authors wish to retract this article "Interference of Mucin1 inhibits the growth of liver cancer cells by inducing mitochondria mediated and death receptor mediated cell apoptosis" by Yongzhen Zhai, Deping Ding, Liya Wei, Enjing Chen, Guohe Feng, published in Tumor Biology, 2014, DOI: 10.1007/s13277-014-2120-9. Concerns were raised on the accuracy of the data presented and as consequence in the results and conclusions of the article for which the authors accept full responsibility. The authors apologize to the readers, reviewers, and editors for publishing this erroneous data.
}

Y. Zhai $\cdot$ L. Wei $\cdot$ E. Chen $\cdot$ G. Feng $(\bowtie)$

Department of Infectious Diseases, Shengjing Hospital of China

Medical University, Shenyang 110004, China

e-mail: feng_guohe@163.com

D. Ding

Department of Infectious Diseases, Taihe Hospital of Hubei

University of Medicine, Shiyan 442000, China 